Advances in Applied Physics, Vol. 2, 2014, no. 1, 27 - 42

HIKARI Ltd, www.m-hikari.com

http://dx.doi.org/10.12988/aap.2014.455

\title{
Surface Plasmonic Contribution to SEIRA and Asymmetrical Band
}

\author{
Hiroshi Nakashima \\ Graduate School of Science and Technology, Hirosaki University \\ Kouji Kita \\ Graduate School of Science, Hirosaki University \\ Yushi Suzuki* \\ Department of Advanced Physics, Hirosaki University \\ Hirosaki, Aomori 036-8561, Japan \\ *Corresponding author \\ Tel./fax: +81 172393556 \\ Copyright (c) 2014 Hiroshi Nakashima et. al. This is an open access article distributed \\ under the Creative Commons Attribution License, which permits unrestricted use, distribu- \\ tion, and reproduction in any medium, provided the original work is properly cited.
}

\begin{abstract}
The Surface Enhanced InfraRed Absorption (SEIRA) band reaches its the maximum intensity and the band shape becomes asymmetric at the just percolated metal thin films. To explain for them, various models (e.g. the Fano model) have been proposed. However, no model can explain them completely. In this paper, we conducted a classical model calculations (Fresnel's formula and the Bruggemann effective medium theory) for SEIRA and asymmetric bands. The calculated SEIRA spectra were good agreement with the experimentally spectra. Therefore, the asymmetrical SEIRA band might not be attributable to Fano model. Moreover, we specifically addressed the relation between effective dielectric constants of a metal-insulator composite thin film and SEIRA band shape change. Although the sign of the real part switched from positive to negative and although the imaginary part became metallic, the asymmetrical band shape did not change. The sign of the real part is
\end{abstract}


expected to be negative and the imaginary part would be metallic if the asymmetrical band shape change results from surface plasmon. Therefore, the asymmetrical band shape might not be attributable to surface plasmon.

Keywords: asymmetrical band, surface-enhanced infrared absorption, SEIRA

\section{Introduction}

Infrared spectroscopy is used in broad fields such as physics, chemistry, biology, and pharmacy [1]. In 1980, the Surface Enhanced InfRared Absorption (SEIRA) effect has been first reported by Hartstein et. al. [2]. This SEIRA effect is used with infrared spectroscopy by which an adsorbates are deposited on a metal thin film to improve sensitivity $[3,4,5,6]$. Using the SEIRA effect, the enhanced absorption intensity is known to increase by some orders of magnitude at the maximum [7]. Therefore, it is useful for biosensors and other devices [8]. By the SEIRA effect, the enhancement factor of the absorption band increases with increasing a discontinuous metal thin film thickness. It becomes the maximum at a percolation film thickness. Furthermore, if the film thickness is more increased, then the enhancement factor of the absorption intensity will decrease. Moreover, near a percolation film thickness, the absorption band shape becomes asymmetric. This asymmetrical absorption band is called a Fano-type band [9], which occurs with SEIRA enhancement effect. Therefore, this asymmetrical band might be related to the SEIRA mechanism. To explain this enhanced absorption and asymmetrical absorption band, various theories have been proposed $[10,11,12]$.

For instance, the model shows that the asymmetrical absorption band occurs because of interactions between vibration modes of adsorbates and electron-hole pairs that are produced in metal thin film [10]. In this model, because the interactions between the electron-hole pair in a metal thin film and the vibration modes of an adsorbate explain the mechanism of enhanced absorption and an asymmetrical absorption band, this effect is restricted to the adsorbates of the first layer, which contact the metal thin film directly. Therefore, for SEIRA and an asymmetrical band of the adsorbates adsorbed directly to the metal thin film, very good results are obtainable. However, an asymmetrical band is observed also by the adsorbate layer, which is separated from the metal thin film by tens of layers or more [13]. Therefore, this model can not fully explain the entire asymmetric absorption band.

In enhancement of absorption intensity, a model that explains enhancement of absorption intensity by physical interactions is proposed [11, 12]. In this model, it is presumed that enhancement of the absorption intensity of an adsorbate will come to occur by the following process: (1) Excitation of 
surface plasmon of the metal thin film by the incident electric field in the UV-Vis-NearIR region. (2) Extension to the MidIR region of the tail of this surface plasmon. (3) Interaction between the electric field, which is enhanced by this surface plasmon tail, and the vibration modes of adsorbates. This is a model of SEIRA effects because of electromagnetic enhancement effects. Therefore, because enhancement effects also affect adsorbates that are not directly adsorbed to the metal thin film surface, this model is consistent with the experimentally obtained result to a greater degree than enhancement model by electron-hole pairs generation. However, in our earlier study [14], if surface plasmon resonance absorption peak in the visible region is blue-shifted and sharpening, then SEIRA is more enhanced. Results showing that the maximum enhancement of SEIRA is observed in a percolation thin film have been obtained from many previous experiments $[15,16,17,18,19]$. In the Osawa model, absorption of a percolation thin film is equivalent to a state in which the surface plasmon absorption peak in the visible region has shifted to the infrared region. Therefore, the result of our experiment and Osawa model that SEIRA results from the red-shifted surface plasmon tail in the infrared region is a contradiction. In addition, other earlier studies[20, 21] have shown that absorption enhancement occurs from the discontinuous metal thin film surface in a short range, and that the morphology of the metal nanoparticles which constitute a discontinuous metal thin film is an important factor.

In this paper, we conducted a classical model calculations (Fresnel's formula and the Bruggemann effective medium theory) for SEIRA and asymmetric bands. From this calculation results, the SEIRA and an asymmetrical band were nicely reproduced. Consequently, the asymmetrical SEIRA band might not be result from the Fano model. Moreover, we specifically addressed the relation between effective dielectric constants of metal-insulator composite thin films and the SEIRA band shape. Although the sign of the real part was switched from positive to negative and although the imaginary part became metallic, the asymmetrical band shape was retained. The sign of real part would be negative and the imaginary part would be metallic if the asymmetrical band shape were result from surface plasmon. Therefore, the asymmetrical band shape might not be attributable to surface plasmon.

\section{Materials and Methods}

\subsection{Experimental methods}

$\mathrm{Si}(100)$ crystals (ca. $20 \times 20 \mathrm{~mm}$ ) were used as substrates of silver films. The crystals were cleaned in an ultrasonically agitated acetone bath. Silver films were deposited at a rate of $1 \mathrm{~nm} / \mathrm{min}$ on $\mathrm{Si}(100)$ substrates at room temperature using electron-beam evaporation in a vacuum chamber evacuated 
at $5 \times 10^{-10}$ Torr. Afterward, each silver film was cooled to $120 \mathrm{~K}$ and was exposed immediately to methanol. Infrared absorption spectra were obtained at normal radiation incidence using an FT-IR spectrophotometer (MB100; ABB Bomen, equipped with liquid nitrogen-cooled MCT detector) with an average of 128 scans at $4 \mathrm{~cm}^{-1}$ resolution.

\subsection{Calculation Model}

The nanoparticle cross section of silver thin film has a hemispheroidal or cylindrical section. The silver nanoparticles are dispersed two-dimensionally on the silicon substrate at random. The effective film thickness of this silver thin film is set to $d_{e f f}=d / f$, where $d$ denotes the mass thickness and $f$ is the area fraction of metal part on a substrate. The silver thin film shape changes as a function of the increase in mass thickness, becoming an island thin film, a percolation thin film, a defective continuous thin film, or a continuous thin film. Because it is extremely difficult to address such a system directly, in this paper, a silver thin film is regarded for simplification as a two-dimensionally dispersed system of cylindrical silver nanoparticles. It is treated as a homogeneous composite layer using effective medium theory. In this effective medium theory, to calculate the dielectric constants of a thin film, there are MaxwellGarnett (MG) theory[22] and Bruggemann (BR) theory[23], which is usually used. Because MG theory can not treat percolation of the metal thin film well and because it can not be used in arbitrary area fractions, BR theory, which can treat both well, is used for this study. From the BR theory, although the equation has two or more solutions, a physically meaningful solution can be determined using the method described in a recent report [24]. The area fraction used for calculation is determined to fulfill the following two conditions: (1) The area fraction increases as the mass thickness increases. (2) The absorption spectrum of a calculation result is the closest to an experimentally obtained result. The area fraction is shown in Table 1.

Up to this point, the model resembles that used in previous studies[12]. Therefore, it is possible to treat the metal thin film system with gaps that are completely filled up with the adsorbates. However, to be calculated in this study, the system also includes additional adsorbates deposited onto the metal thin film, presenting a gap that is filled up completely with adsorbates. In SEIRA band calculation, it is necessary to account for the amount of adsorbates on the metal thin film accurately. Therefore, we extend the model below. The actual deposition process of methanol might be uniform. However, in this paper, it is approximated for simplification that methanol is deposited preferentially on crevices between islands. From this approximation, at a certain exposure, the exposure of methanol deposited on crevices between islands differs from the real state. An earlier study $[25,26]$ revealed that the adsorbates 
deposited on the crevices between islands are mainly sensitive to the SEIRA effect. Therefore, low exposure might produce error in calculation of an early stage. However, because we specifically addressed the band shape in this paper, this point is not examined. A schematic diagram for calculation is presented in Fig. 1. In addition to this model, the hemispheroidal nanoparticle model and the spheroidal nanoparticle model are calculated. However, the calculation result is almost identical to the cylindrical model. Therefore, the calculation result of the physically simplest model is used. In this model, when the exposure of adsorbate is less than the exposure completely filling up the crevices between islands, it is calculated as a four-layer system of substrate/metaladsorbate composite/metal-vacuum composite/vacuum (Fig. 1(b)). When there is greater exposure of adsorbate than the exposure completely filled up with the crevices between islands, it is calculated as a four-layer system of substrate/metal-adsorbate composite/adsorbate/vacuum (Fig. 1(c)). Therefore, to calculate the absorption spectrum of this system, the formula of transmittance of a four-layer system is used. The formula of the transmittance of a three-layer system is used for calculation of a spectrum in case an adsorbate is

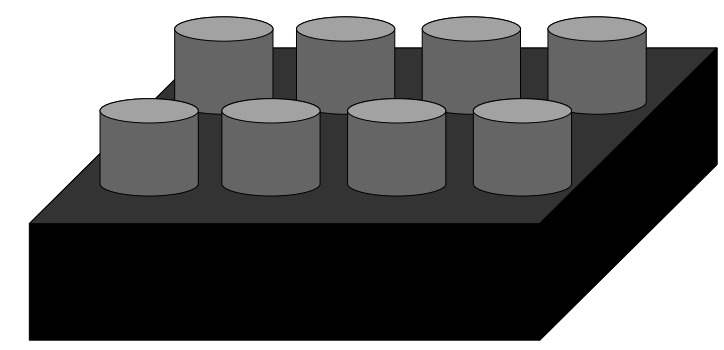

(a)

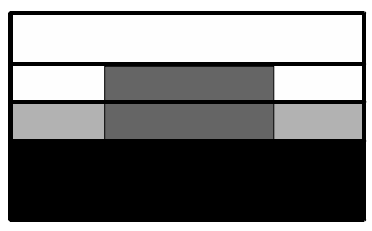

(b)

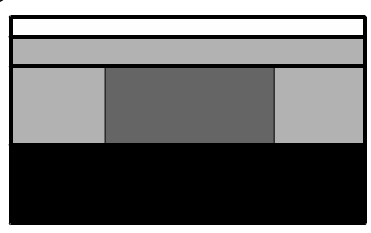

(c)

Figure 1: Schematic diagrams of the computational model. (a) Schematic diagram of computational model. In this model, silver islands are regarded as cylindrical, and are treated as a homogenized composite layer using BR theory. (b) Side view of the system of a small amount of methanol deposited rather than filling crevices. In the (b) state, the treatment is sequential from the substrate side: Si/silver, methanol composite/silver, vacuum composite/vacuum. (c) Side view of system of large amount of methanol deposited rather than filling crevices. In the (c) state, the treatment is sequential from the substrate side: Si/silver, methanol composite/methanol/vacuum. 
Table 1: The mass thickness of silver, the area fraction of silver islands used for calculation, and exposure of methanol required for filling of crevices between silver islands. The first column shows the sample number of respective samples. The second shows the mass thickness of silver of respective samples. The third presents the area fraction used for calculation of effective medium theory, determined such that it will fulfill the following conditions. (a) The area fraction increases as the mass thickness increases. (b) The shape of the spectrum of the calculation result shows best agreement with experimentally obtained results. The fourth column shows the exposure of methanol needed for filling of the crevices between silver islands of respective samples.

\begin{tabular}{cccc}
\hline Sample Number & Mass Thickness [nm] & Area Fraction & Exposure [Layer] \\
\hline 1 & 1 & 0.186 & 12.50 \\
2 & 3 & 0.380 & 13.98 \\
3 & 5 & 0.460 & 16.77 \\
4 & 7 & 0.479 & 21.75 \\
5 & 8 & 0.511 & 21.87 \\
6 & 9 & 0.529 & 22.89 \\
7 & 10 & 0.546 & 23.76 \\
\hline
\end{tabular}

deposited directly on a substrate, and the system of substrate/metal-vacuum composite/vacuum is used as a background spectrum.

The formulae for transmittance of a three-layer system and a four-layer system are derived using the concept of resultant wave described in an earlier report [27], Snell's law, and Fresnel's formula. The formula of transmittance and reflectance of a four-layer system is presented below, where the refractive index of the medium of the $j$-th layer is $n_{j}$, an extinction coefficient is $k_{j}$ and a complex refractive index is $\hat{n_{j}}=n_{j}+i k_{j}$. The $\boldsymbol{E}_{\mathbf{0}}^{-}$and $\boldsymbol{E}_{\mathbf{3}}^{+}$that are necessary for calculating the reflectance and transmittance of a four-layer system are presented below.

$$
\begin{aligned}
& \boldsymbol{E}_{\mathbf{0}}^{-}=\frac{r_{1}+r_{2} e^{-2 i \delta_{1}}+r_{3} e^{-2 i\left(\delta_{1}+\delta_{2}\right)}+r_{1} r_{2} r_{3} e^{-2 i \delta_{2}}}{1+r_{1} r_{2} e^{-2 i \delta_{1}}+r_{1} r_{3} e^{-2 i\left(\delta_{1}+\delta_{2}\right)}+r_{2} r_{3} e^{-2 i \delta_{2}}} \boldsymbol{E}_{\mathbf{0}}^{+} \\
& \boldsymbol{E}_{\mathbf{3}}^{+}=\frac{t_{1} t_{2} t_{3} e^{-i\left(\delta_{1}+\delta_{2}\right)}}{1+r_{1} r_{2} e^{-2 i \delta_{1}}+r_{1} r_{3} e^{-2 i\left(\delta_{1}+\delta_{2}\right)}+r_{2} r_{3} e^{-2 i \delta_{2}}} \boldsymbol{E}_{\mathbf{0}}^{+} \\
& \delta_{j}=\frac{2 \pi \hat{n_{j}} d_{j}}{\lambda}
\end{aligned}
$$

In those equations, the thickness of medium 1 is $d_{1}$, the thickness of medium 2 is $d_{2}$, the amplitude of the electric field vector of incident light in medium 0 is $\boldsymbol{E}_{\mathbf{0}}^{+}$, the amplitude of the electric field vector of reflected light in medium 0 is $\boldsymbol{E}_{\mathbf{0}}^{-}$, the amplitude of the electric field vector of transmitted light in medium 3 is $\boldsymbol{E}_{\mathbf{3}}^{+}$, and the phase change on traversing the $j$-th medium is $\delta_{j}$. The formulae of transmittance and reflectance of four-layer system obtained are as follows.

$$
R=\frac{E_{0}^{-} \overline{E_{0}^{-}}}{E_{0}^{+}}
$$




$$
T=\frac{\hat{n_{3}}}{\hat{n_{0}}} \frac{\boldsymbol{E}_{3}^{+} \overline{\boldsymbol{E}_{3}^{+}}}{\boldsymbol{E}_{\mathbf{0}}^{+} \overline{\boldsymbol{E}_{\mathbf{0}}^{+}}}
$$

Because it is considered that each silver nanoparticle of a silver thin film has optical constants of the silver of bulk [28], we use the data of the silver of bulk [29] for calculations presented herein. The used optical constants of methanol are obtained from the absorption spectrum of methanol in the case of without silver thin film using parameters in Ref. [30] and Ref. [31]. This optical constants of methanol is almost same to that described in an earlier report [31]. The dielectric constants of vacuum are set to $\hat{\epsilon_{v a c}}=(1.0,0.0)$ and the optical constants of the silicon substrate to $\hat{\epsilon_{S i}}=(11.70,0.0)[29]$.

\section{Results and Discussion}

\subsection{Experimental SEIRA Spectra of Methanol}

Infrared absorption spectra of the methanol adsorbed onto the silver thin film evaporated on the silicon substrate measured experimentally as a function of exposure of methanol and silver mass thickness are shown in Fig. 2. Moreover, an SEM image of the sample corresponding to these spectra is shown in Fig. 3. The exposure of methanol corresponding to each spectrum of Fig. 3 is presented in Table 2. When the mass thickness of a silver film is decreased, the spectrum shape becomes almost identical (Figs. 2(a)-2(c)). The absorption intensity of a spectrum of a silver film increases as a function of the mass thickness, where a silver film is almost an island thin film (Figs. 3(a)-3(c)). When the mass thickness of a silver film is increased, then the shape of a spectrum becomes completely asymmetrical (Figs. 2(d), 2(e)). This asymmetrical band has been described in an earlier report [13]. Probably, in past studies $[32,33,34,35]$, the existence of Fano Resonance was assumed from this asymmetrical band. The absorption intensity of this sample is the maximum in the sample in this study, and it is starting percolation of the silver film (Figs. 3(d) and $3(\mathrm{e}))$. However, for mass thickness of $9 \mathrm{~nm}$, the asymmetrical band is remarkably absent (Fig. 2(f)). The absorption band of this sample has weak intensity and a sharp shape. Moreover, the film morphology resembles that of a continuous film (Fig. 3(f)). When the mass thickness is $10 \mathrm{~nm}$, the absorption intensity is weak. Remarkably, the asymmetrical band shape is inverted (Figs. 2(d), 2(e), 2(g)). For the disappearance and inversion of asymmetrical band, the report in this paper is the first. The spectrum of Fig. 2(g) is noisier than those of other spectra. This originates in the difference of background transmittance of the sample of each mass thickness because the background transmittance of the sample of Fig. 2(a) to Fig. 2(f) with little noise is from about 1 to 0.2 . However, Fig. 2(g) has about 0.05. Results show that the 
absolute intensity of the signal to a noise is small, and the spectra of Fig. 2(g) become noisy.

\subsection{Comparison of Calculated and Measured SEIRA Spectra}

The calculated spectra are shown in Fig. 4 with corresponding experimental spectra. The methanol exposure corresponding to each spectrum of Fig. 4 is presented in Table 2. The calculated spectra show good agreement with experimental spectra by the classical model (Fresnel's formula and BR theory) calculated using the same conditions. Consequently, an asymmetrical band might not be based only on a Fano effect. Presuming that the cause of an asymmetrical band is not the Fano effect, it is necessary to consider what caused the asymmetrical band. At present, generally an asymmetrical band is thought to originate in Surface Plasmon (SP) $[12,36]$. SP is collective oscillation of the free-electron localized in the surface. Therefore, it will occur when a silver thin film is macroscopically in a metallic state. Therefore, if an asymmetrical band originates in SP, then the appearance of an asymmetrical band and sign switching of the dielectric constants of a silver thin film is expected to occur simultaneously. To verify this point, the complex dielectric constants of silver-methanol composite thin film calculated with BR theory are shown in Fig. 5. In Fig. 4, the spectra for the sample of mass thickness $7 \mathrm{~nm}$ and $8 \mathrm{~nm}$ have similar asymmetrical shape and absorption intensity. However, the signs of the real part of the effective dielectric constants differ from each other: the $7 \mathrm{~nm}$ sample is positive; the $8 \mathrm{~nm}$ sample is negative. Simultaneously with the sign switching, the crossover of inclination of both the real part and imaginary part of a complex dielectric constants has occurred. This crossover has proved that the dielectric function of the silver thin film changed from insulator-like to metal-like with the sign switching of the real part of complex dielectric constants $[37,38,39]$. However, although the silver thin film is changing from an insulator-like state (positive) to a metal-like state (negative), the asymmetrical band is kept. In other words, the appearance of an asymmetrical band and the change of SP state are not simultaneous. Therefore, the main cause of the asymmetrical absorption band might not be SP.

Next, we specifically examined absorption enhancement. To consider absorption enhancement, the Enhancement Factor (EF) is defined as a ratio of the absorption intensity with silver and without silver. In the experimentally obtained result, when the methanol exposure is about 50 layers, EFs were 2.5 $(1 \mathrm{~nm}), 5.8(3 \mathrm{~nm}), 16.0(5 \mathrm{~nm}), 39.2(7 \mathrm{~nm}), 34.2(8 \mathrm{~nm}), 9.8(9 \mathrm{~nm})$, and $3.0(10 \mathrm{~nm})$ in each sample. In the calculation result obtained using a classical model, when the methanol exposure is about 50 layers, EFs were $1.2(1 \mathrm{~nm})$, 
Table 2: Exposure of methanol corresponding to each measured spectrum to the sample of each mass thickness. The unit of exposure is layers.

\begin{tabular}{|c|c|c|c|c|c|c|c|c|c|}
\hline & & \multicolumn{6}{|c|}{ Silver Thin Film Mass Thickness } & \multicolumn{2}{|l|}{$\mathrm{nm}]$} \\
\hline & & no-Ag & 1 & 3 & 5 & 7 & 8 & 9 & 10 \\
\hline \multirow{26}{*}{ 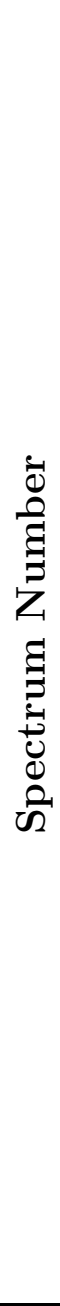 } & 1 & 11.22 & 1.02 & 1.76 & 3.26 & 1.28 & 1.68 & 1.96 & 1.29 \\
\hline & 2 & 15.64 & 2.66 & 3.27 & 4.98 & 1.85 & 2.60 & 3.48 & 2.26 \\
\hline & 3 & 24.39 & 3.88 & 5.02 & 6.99 & 3.38 & 3.80 & 5.46 & 6.68 \\
\hline & 4 & 58.14 & 5.01 & 6.82 & 8.80 & 6.89 & 4.80 & 8.80 & 17.07 \\
\hline & 5 & 100.53 & 6.85 & 10.71 & 11.34 & 9.40 & 7.78 & 11.65 & 23.98 \\
\hline & 6 & 158.94 & 8.73 & 16.26 & 15.69 & 11.16 & 10.73 & 16.84 & 50.03 \\
\hline & 7 & 282.31 & 10.41 & 20.60 & 20.48 & 16.48 & 14.69 & 25.69 & 70.14 \\
\hline & 8 & 376.49 & 12.54 & 25.68 & 26.85 & 21.55 & 21.22 & 34.58 & 111.49 \\
\hline & 9 & & 16.22 & 31.43 & 32.50 & 28.87 & 29.76 & 41.82 & 150.89 \\
\hline & 10 & & 20.89 & 36.68 & 38.11 & 37.56 & 34.44 & 49.35 & 219.54 \\
\hline & 11 & & 26.54 & 41.37 & 41.74 & 44.70 & 39.81 & 58.06 & 362.00 \\
\hline & 12 & & 32.30 & 45.75 & 46.73 & 49.73 & 45.51 & 69.79 & 681.24 \\
\hline & 13 & & 38.59 & 50.50 & 51.14 & 56.09 & 51.28 & 89.62 & \\
\hline & 14 & & 43.86 & 55.56 & 60.96 & 63.18 & 59.19 & 112.88 & \\
\hline & 15 & & 52.25 & 60.61 & 72.74 & 70.56 & 66.77 & 155.59 & \\
\hline & 16 & & 66.68 & 67.93 & 86.03 & 78.54 & 77.80 & 241.42 & \\
\hline & 17 & & 84.79 & 81.15 & 109.02 & 86.75 & 103.79 & 381.59 & \\
\hline & 18 & & 96.98 & 101.29 & 137.26 & 109.38 & 149.87 & & \\
\hline & 19 & & 112.58 & 139.52 & 171.04 & 149.90 & 249.51 & & \\
\hline & 20 & & 122.31 & 217.02 & 220.84 & 215.94 & 394.28 & & \\
\hline & 21 & & 136.14 & 335.15 & 292.82 & 339.42 & 766.26 & & \\
\hline & 22 & & 145.58 & & 355.00 & 738.41 & & & \\
\hline & 23 & & 167.29 & & 749.10 & & & & \\
\hline & 24 & & 183.25 & & & & & & \\
\hline & 25 & & 222.14 & & & & & & \\
\hline & 26 & & 282.42 & & & & & & \\
\hline
\end{tabular}




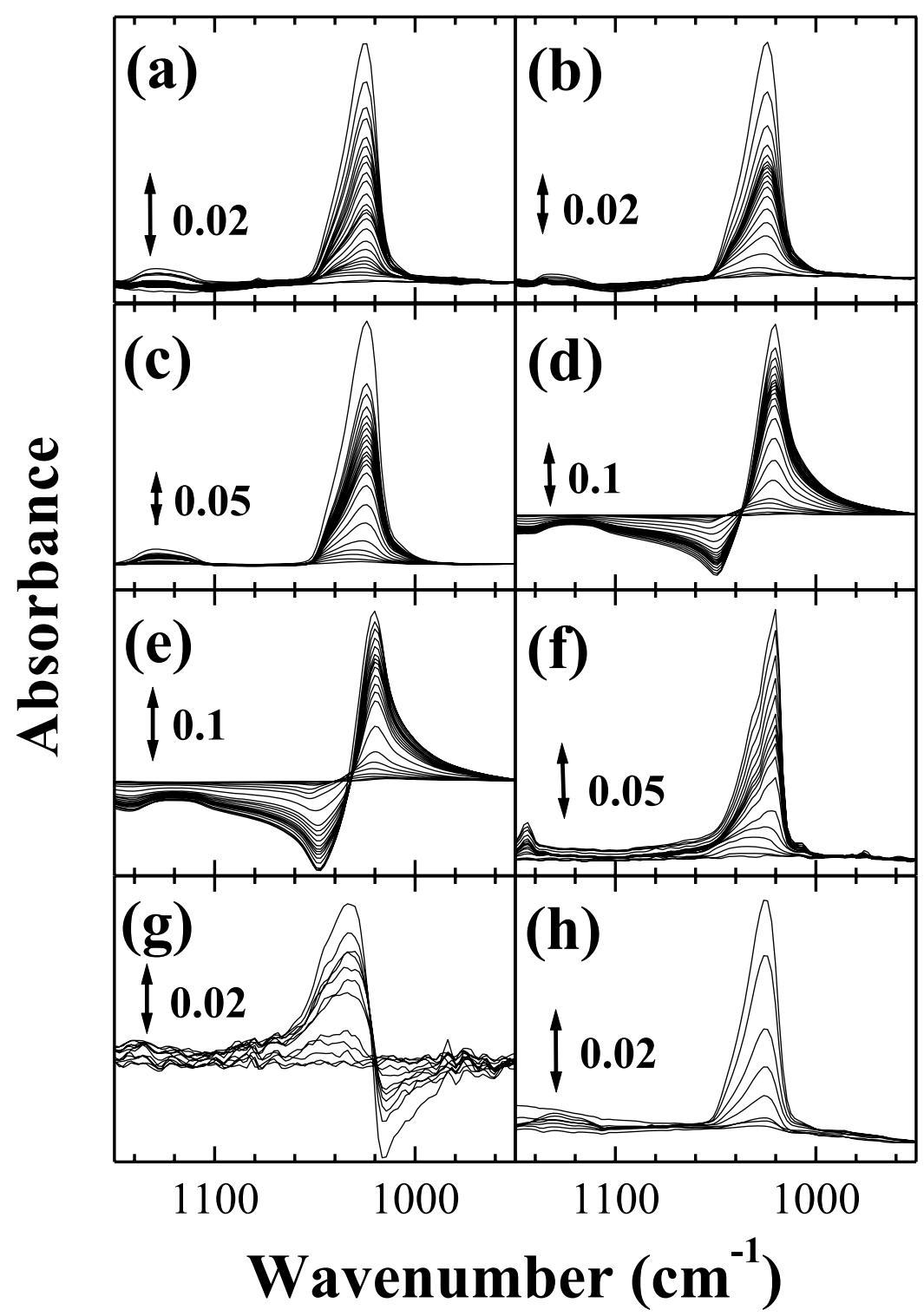

Figure 2: Measured infrared absorption spectra of methanol deposited on a silver thin film on a silicon substrate. The mass thicknesses of respective samples are the following: (a) $1 \mathrm{~nm}$, (b) $3 \mathrm{~nm}$, (c) $5 \mathrm{~nm}$, (d) $7 \mathrm{~nm}$, (e) $8 \mathrm{~nm}$, (f) $9 \mathrm{~nm},(\mathrm{~g}) 10 \mathrm{~nm}$, and (h) no-Ag. Exposure to methanol corresponding to each spectrum is presented in Table 2. For reference, the measured spectra of silver thin film on a silicon substrate sample were used. 



Figure 3: SEM images of silver thin films deposited on a silicon substrate. Mass thicknesses of respective samples are the following: (a) $1 \mathrm{~nm}$, (b) $3 \mathrm{~nm}$, (c) $5 \mathrm{~nm}$, (d) $7 \mathrm{~nm}$, (e) $8 \mathrm{~nm}$, (f) $9 \mathrm{~nm}$, and (g) $10 \mathrm{~nm}$. The scales are shown in respective images. 


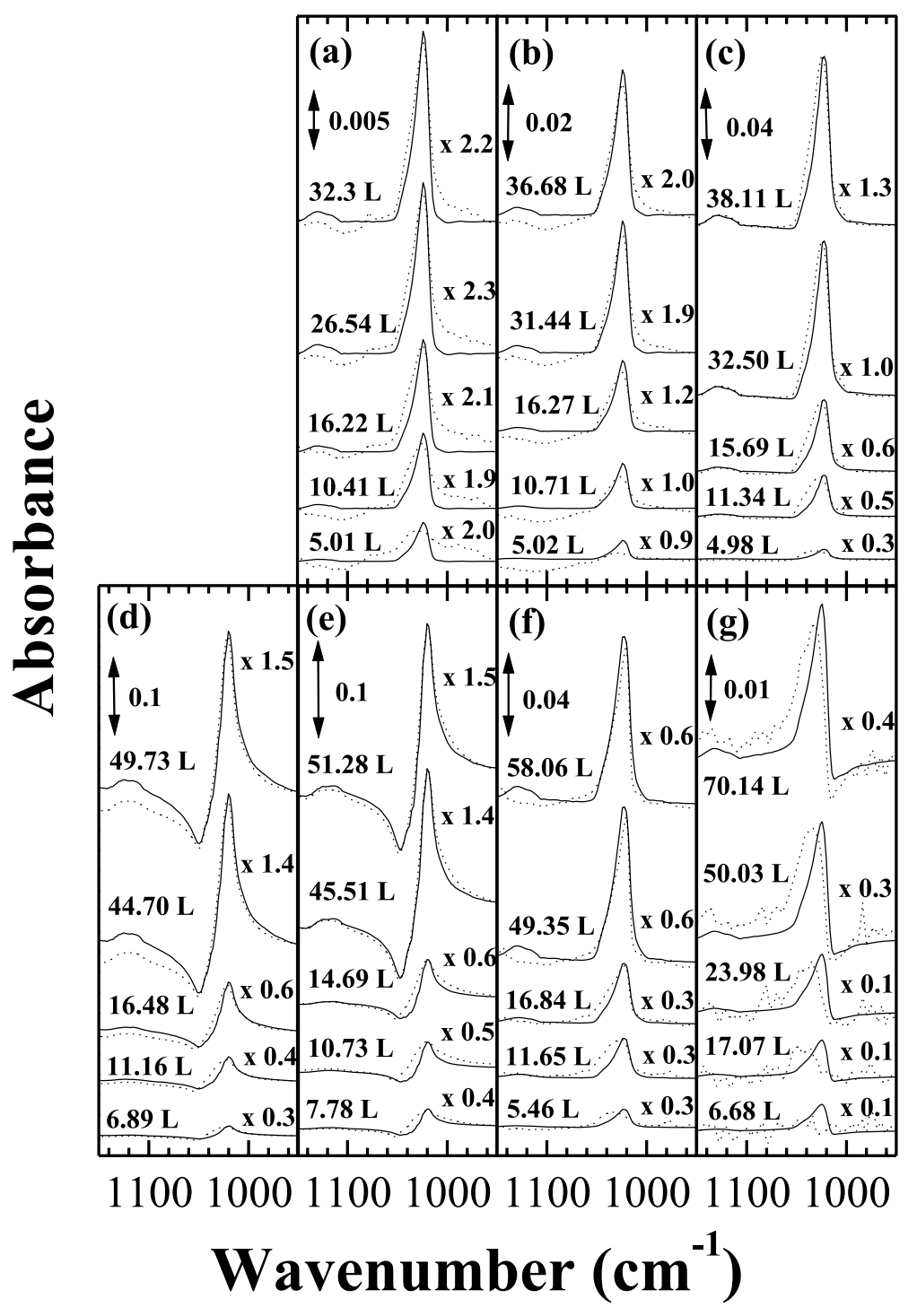

Figure 4: Comparison plot of experimental and calculated infrared absorption spectra of methanol deposited on a silver thin film on a silicon substrate. Solid lines show calculated spectra and dotted lines are measured spectra. Mass thicknesses of respective samples are the following: (a) $1 \mathrm{~nm}$, (b) $3 \mathrm{~nm}$, (c) $5 \mathrm{~nm}$, (d) $7 \mathrm{~nm}$, (e) $8 \mathrm{~nm}$, (f) $9 \mathrm{~nm}$, and (g) $10 \mathrm{~nm}$. Exposure of methanol corresponding to each spectrum is shown in the left-hand side of the figure. The coefficient was applied to set the scale of the experimental and a calculated spectrum. The coefficient is shown on the right-hand side in the figure. 




Figure 5: Complex dielectric constants of a composite layer of silver and methanol calculated using BR theory. The left-hand side is the real part. The right-hand side is the imaginary part. The mass thicknesses of respective samples are (a) $1 \mathrm{~nm}$, (b) $3 \mathrm{~nm}$, (c) $5 \mathrm{~nm}$, (d) $7 \mathrm{~nm}$, (e) $8 \mathrm{~nm}$, (f) $9 \mathrm{~nm}$, and (g) $10 \mathrm{~nm}$. The area fraction used for calculation of each complex dielectric constant is presented in Table 1. 
$2.6(3 \mathrm{~nm}), 9.4(5 \mathrm{~nm}), 22.3(7 \mathrm{~nm}), 18.5(8 \mathrm{~nm}), 16.2(9 \mathrm{~nm})$, and $9.1(10$ $\mathrm{nm})$ in each sample. These results demonstrate that the absorption intensities of measured spectra are about two times higher than those of calculated spectra (without the samples of $9 \mathrm{~nm}$ and $10 \mathrm{~nm}$ ). In other words, the contribution of the enhancement effect, which is not considered in present classical model calculation, is about two times at maximum. It is also noteworthy that the maximum value of the EF was observed in the insulator-like discontinuous metal thin film. This result shows that the main enhancement effect of infrared absorption enhancement is included in the classical formula used in this calculation, and that enhancement is about 20 times at the maximum. In other studies [12], it is presumed that this enhancement effect originates in SP. However, according to the results presented in Fig. 5 and the past report [14], the main enhancement effect of SEIRA might not be a SP. The Square Columnar Model [40] remains as a hypothesis related to the enhancement mechanism independent of SP. Detailed elucidation of this enhancement mechanism remains as a task for future research.

\section{Conclusion}

As described in this paper, to study a SEIRA phenomenon and an asymmetrical absorption band, we calculated a spectrum through numerical computation using a classical model (Fresnel's formula, BR theory). The SEIRA and asymmetrical absorption band were nicely reproduced by the classical model. In the past, although Fano models were proposed for the asymmetrical line shape, even if a Fano model were not considered, an asymmetrical absorption band might occur with an SEIRA phenomenon. In addition, the appearance of asymmetrical band shape and the changes of dielectric constants of metal thin films (insulator-like to metal-like) were not simultaneous. Consequently, the asymmetrical band shape might not be attributable to surface plasmon. Moreover, SEIRA enhancement has been reproduced by the classical model. The differences of intensity between experimentally obtained results and calculated results are about two times. Therefore, the maximum contribution of non-classical model (e.g., Fano model and surface plasmon) in SEIRA enhancement is about two times. Furthermore, results show that the dielectric constants of the metal thin film are insulator-like when the SEIRA enhancement factor is the maximum. From this result, we infer that the main enhancement effect of SEIRA might not be attributable to surface plasmon.

\section{References}

[1] M. Osawa, Topics in Appl. Phys. 81 (2001) 163. 
[2] A. Hartstein, J.R. Kirtley, J.C. Tsang, Phys. Rev. Lett. 45 (1980) 201.

[3] A. Hatta, Y. Suzuki, W. Suetaka, Appl. Phys. A 35 (1984) 135.

[4] Y. Suzuki, S. Shimada, A. Hatta, W. Suetaka, Surf. Sci. 219 (1989) 595.

[5] D.A. Heaps, P.R. Griffiths, Vibrational Spectroscopy 42 (2006) 45.

[6] R.F. Aroca, D.J. Ross, C. Domingo, Appl. Spectroscopy 58 (2004) 324.

[7] Y. Suzuki, M. Osawa, A. Hatta, W. Suetaka, Appl. Surf. Sci. 33/34 (1988) 875.

[8] C.W. Brown, Y. Li, J.A. Seelenbinder, P. Pivarnik, A.G. Rand, S.V. Letcher, O.J. Gregory, M.J. Platek, Anal. Chem. 70 (1998) 2991.

[9] U. Fano, Phys. Rev. 124 (1961) (6) 1866.

[10] D.C. Langreth, Phys. Rev. Lett. 54 (1985) 126.

[11] M. Osawa, Bull. Chem. Soc. Jpn. 70 (1997) 2861.

[12] A. Priebe, M. Sinther, G. Fahsold, A. Pucci, J. Chem. Phys. 119 (2003) 4887.

[13] Y. Suzuki, S. Goto, H. Umetsu, Eur. Phys. J. D 33 (2005) 201.

[14] Y. Suzuki, H. Makanae, H. Kudo, T. Miyanaga, T. Nanke, T. Kobayashi, Appl. Phys. A 78 (2004) 335.

[15] A.E. Bjerke, P.R. Griffiths, Appl. Spectroscopy 56 (2002) 101275.

[16] M. Lust, A. Priebe, G. Fahsold, A. Pucci, Surf. Interface Anal. 33 (2002) 6487.

[17] D.A. Heaps, P.R. Griffiths, Vibrational Spectroscopy 42 (2006) 145.

[18] D. Enders, A. Pucci, Appl. Phys. Lett. 88 (2006) 184104.

[19] D. Enders, T. Nagao, A. Pucci, T. Nakayama, M. Aono, Phys. Chem. Chem. Phys. 13 (2011) 4935.

[20] T. Tanabe, T. Morisato, Y. Suzuki, Y. Matsumoto, T. Wadayama, A. Hatta, Vibrational Spectroscopy 18 (1998) 141.

[21] Y. Suzuki, K. Kita, N. Matsumoto, Phys. Low-Dim. Struct.1/2 (2001) 1.

[22] J.C. Maxwell-Garnett, Phil. Trans. Roy. Soc. A203 (1904) 385, A205 (1906) 237. 
[23] D.A.G. Bruggemann, Ann. Phys. (Leipzig) 24 (1935) 636.

[24] R. Jansson, H. Arwin, Optics. Comm. 106 (1994) 133.

[25] Y. Suzuki, H. Seki, T. Inamura, T. Tanabe, T. Wadayama, A. Hatta, Surf. Sci. 427-428 (1999) 136-140.

[26] Y. Suzuki, H. Seki, T. Inamura, T. Tanabe, T. Wadayama, A. Hatta, Surf. Sci. 433-435 (1999) 261-266.

[27] O.S. Heavens, Optical Properties of Thin Solid Films, Dover Publications Inc., New York, 1992 chapter 4.

[28] Y. Suzuki, Y. Sumi, K. Kita, T. Miyanaga, K. Sagisaka, Surf. Interface Anal. 38 (2006) 1296.

[29] E.D. Palik, Handbook of Optical Constants of Solids, Academic Press, San Diego, 1997 Vol.1.

[30] J.J. Vijayaa, L.J. Kennedy, G. Sekaran, B. Jeyaraj, K.S. Nagaraja, J. Hazardous Materials 153 (2008) 1-2 767.

[31] J.E. Bertie, S.L. Zhang, Can. J. Chem. 70 (1992) 520.

[32] O. Krauth, G. Fahsold, A. Pucci, J. Chem. Phys. 110 (1999) 3113.

[33] O. Krauth, G. Fahsold, N. Magg, A. Pucci, J. Chem. Phys. 113 (2000) 6330 .

[34] A. Priebe, G. Fahsold, A. Pucci, Surf. Sci. 482 (2001) (85) 190.

[35] C.X. Wu, H. Lin, Y.J. Chen, W.X. Li, S.G. Sunb, J. Chem. Phys. 121 (2004) 1553.

[36] T.R. Jensen, R.P. Van Duyne, S.A. Johnson, V.A. Maroni, Applied Spectroscopy 54 (2000) 3371.

[37] G. Fahsold, A. Bartel, O. Krauth, N. Magg, A. Pucci, Phys. Rev. B 61 (2000) 14108.

[38] F. Meng, A. Pucci, phys. stat. sol. (b) 244 (2007) 3739.

[39] M. Hövel, B. Gompf, M. Dressel, Thin Solid Films 519 (2011) 2955.

[40] Y. Suzuki, K. Kita, N. Matsumoto, Appl. Phys. A 77 (2003) 613.

\section{Received: May 1, 2014}

